\title{
Bunching transition in a time-headway model of a bus route
}

\author{
Takashi Nagatani \\ Division of Thermal Science, Department of Mechanical Engineering, Shizuoka University, Hamamatsu 432-8561, Japan
}

(Received 25 September 2000; published 22 February 2001)

\begin{abstract}
A time-headway model is presented to mimic bus behavior on the bus route. The motion of a bus is described in terms of the time headway between its bus and the bus in front. We study the bunching behavior of buses induced by interacting with other buses and passengers. It is shown that the dynamical phase transitions among the inhomogeneous bunching phase, the homogeneous free phase, the coexisting phase, and the homogeneous congested phase occur with varying the initial time headway. We study the effect of not stopping at bus stops on the time-headway profile. It is found that the bunching transition lines are consistent with the neutral stability curves obtained by the linear stability analysis.
\end{abstract}

DOI: 10.1103/PhysRevE.63.036115

PACS number(s): 05.70.Fh, 64.60.-i, 89.40.+k

\section{INTRODUCTION}

Recently, traffic problems [1-4] have attracted much attention in the field of nonequilibrium statistical mechanics. Traffic flow is a kind of many-body system of strongly interacting cars. The traffic flow has been studied by various models: the cellular automaton models, the car following models, the gas kinetic models, and the hydrodynamic models [5-13]. Recent studies reveal physical phenomena such as the nonequilibrium phase transitions and the nonlinear waves [14-26]. It has been shown that the jamming transitions are very similar to the conventional phase transitions and critical phenomena [27,28].

Very recently, the bus route model has been proposed to investigate the bus behavior on a bus route with the use of the cellular automaton [29]. The bus route mode is also a typical many-body system. If a bus is delayed by some fluctuation, the time headway (gap) between it and its predecessor becomes larger than the initial time headway because this bus has to pick up more passengers than the initial value. During the period of delay, more passengers will be waiting for the bus. As a result, the bus will get further delayed. The slowly moving delayed bus will slow down the buses behind it. It has been found that the bunching transition between an inhomogeneous jammed phase (where the buses bunch together) and a homogeneous phase occurs with varying the bus density. The dynamical phase transition and scaling behavior of the time headway have been studied.

The bus route dynamics is closely related to the traffic flow dynamics in one dimension. However, the studies of the bus route problem are very scarce [29]. To know the properties of the buses on the route is important in our life. The bus route problem is also interesting from the point of view of nonequilibrium phase transitions. It is sometimes observed that when a bus is highly delayed, the bus passes the bus stop without stopping. Furthermore, the nonstopping bus increases its speed to reduce the delay. It is little known how the nonstopping and speed up of the delayed bus effects the bus behavior. The reduction of the delay is very important to the public.

The traffic flow systems have been studied using various models. The car-following models have some advantages for modeling and analysis. In the car-following model, it is easy to take into account the local dynamics of cars. The linear stability analysis can be applied to the basic equation and the jamming transition curve can be calculated by the neutral stability line. On the other hand, the bus route system has been studied only by the cellular automaton model [29]. The other models are unknown in literature.

In this paper we present the time-headway model to mimic the behavior of buses on the route. We study the time-headway behavior of buses by the use of both simulation and linear stability analysis. We show that the bunching transition occurs in the time-headway model. We investigate the effect of not stopping at the bus stop on the timeheadway profile and the bunching transition.

\section{MODELS}

We modify the car-following model of traffic to describe the bus motion in terms of the time headway. First, we consider the basic model with stopping at the bus stop. Each bus stops at all the bus stops. It is assumed that the bus behind cannot pass over the bus ahead. We now define the basic model for the bus route. The model is defined on a onedimensional (1D) lattice with periodic boundary conditions. Each lattice site is labeled with a number $m$ running from 1 to $M$. A site represents a bus stop. Buses move on the $1 \mathrm{D}$ lattice. Each bus is labeled with a number $j$ running from 1 to $N$. The distance between bus stops $m-1$ and $m$ is set by $L_{m-1}$. The model is illustrated schematically in Fig. 1 . The mean velocity of bus $j$ between bus stops $m-1$ and $m$ is defined by $v_{j}(m-1)$. The arrival time $t_{j}(m)$ of bus $j$ at bus stop $m$ is given by

$$
t_{j}(m)=t_{j}(m-1)+\frac{L_{m-1}}{v_{j}(m-1)} .
$$

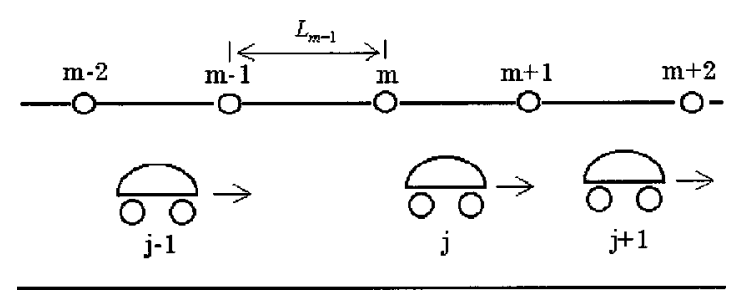

FIG. 1. Schematic illustration of the bus route model. 
We define the time headway of bus $j$ at bus stop $m$ as follows:

$$
\Delta t_{j}(m)=t_{j}(m)-t_{j+1}(m)
$$

where $t_{j+1}(\mathrm{~m})$ is the arrival time of bus $j+1$ in front of bus $j$ at bus stop $m$. From Eqs. (1) and (2), one obtains the equation of motion for bus $j$ in terms of time headway

$$
\Delta t_{j}(m)=\Delta t_{j}(m-1)+L_{m-1}\left[\frac{1}{v_{j}(m-1)}-\frac{1}{v_{j+1}(m-1)}\right] .
$$

Generally, a bus driver operates his bus in such a manner that his velocity increases or decreases according as the time headway is large or small. We assume that the mean velocity of bus $j$ at bus stop $m$ depends only on the time headway of bus $j$ at bus stop $m$. The mean velocity is given by the following operation velocity function

$$
v_{j}(m)=V\left(\Delta t_{j}(m)\right),
$$

where $V\left(\Delta t_{j}(m)\right)$ is the function of only time headway $\Delta t_{j}(m)$. The operation velocity is similar to the optimal velocity function in the car-following model for traffic flow [27].

Let us include the characteristic of the bus route in the operation velocity function (4). We adopt the following operation velocity:

$$
\begin{aligned}
V\left(\Delta t_{j}(m)\right)= & v_{\min }+\frac{\left(v_{\max }-v_{\min }\right)}{4} \\
& \times\left[\tanh \left(\Delta t_{j}(m)-t_{c}\right)+\tanh \left(t_{c}\right)\right] \\
& \times\left[1+\exp \left(-\lambda \Delta t_{j}(m)\right],\right.
\end{aligned}
$$

where $\lambda$ is the rate of arrival of passengers, $v_{\min }$ is the minimal velocity, $v_{\max }$ is the maximal velocity in the limit of $\lambda$ $=0$, and $t_{c}$ is the desired time headway. When $\lambda=0$, the operation velocity function (5) is consistent with the optimal velocity function of the traffic model except for $v_{\min } \neq 0$ and the replacement of the distance headway with the time headway. The operation velocity function (5) has the following properties: (1) in the limit of $\lambda=0$, the velocity increases monotonically with the time headway and (2) the velocity decreases with increasing time headway for small $\lambda$ and large time headway. The property (1) reflects the fact that when the bus behind catches up with the delayed bus, it should slow down in order to avoid a collision. The property (2) reflects the fact that the bus is delayed more and more with increasing the gap size (time headway) in front of that bus because the delayed bus should pick up more passengers. Figure 2 shows the plot of Eq. (5) against time headway for $\lambda=0.2, v_{\min }=2.0, v_{\max }=4.0$, and $t_{c}=2.0$. Thus the bus route problem is reduced to its simplest form. The essential features are maintained.

We take into account no stopping of the delayed bus. If a bus is very delayed, the bus should pass over the bus stop without stopping at the bus stop. It is assumed that a bus trend to pass over the bus stop when the time headway of the

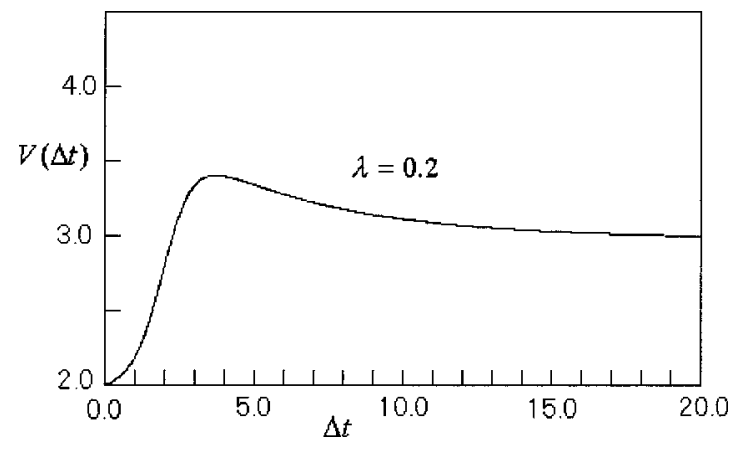

FIG. 2. Plot of the operation velocity $V(\Delta t)$ against time headway $\Delta t$ for $\lambda=0.2, v_{\min }=2.0, v_{\max }=4.0$, and $t_{c}=2.0$.

bus is larger than the critical value $t_{s}$. If the bus passes over the bus stop without stopping, the passengers waiting at the bus stop take the next bus. Then, we adopt the modified operation velocity function:

$$
\begin{aligned}
V\left(\Delta t_{j}(m)\right)= & v_{\min }+\frac{\left(v_{\max }-v_{\min }\right)}{4} \\
& \times\left[\tanh \left(\Delta t_{j}(m)-t_{c}\right)+\tanh \left(t_{c}\right)\right] \\
& \times\left\{1+\exp \left[-A\left(\Delta t_{j}(m), \Delta t_{j+1}(m)\right)\right]\right\},
\end{aligned}
$$

with

$$
\begin{aligned}
A\left(\Delta t_{j}(m), \Delta t_{j+1}(m)\right) \\
=\lambda\left[\Delta t_{j}(m)-\Delta t_{j}(m)\left\{1+\tanh b\left(\Delta t_{j}(m)-t_{s}\right)\right\} / 2\right. \\
\left.\quad+\Delta t_{j+1}(m)\left\{1+\tanh b\left(\Delta t_{j+1}(m)-t_{s}\right)\right\} / 2\right] . \quad(7)
\end{aligned}
$$

When $\Delta t_{j}(m)<t_{s}$ and $b \rightarrow \infty$, the second term of Eq. (7) becomes zero. If $\Delta t_{j}(m)>t_{s}$ and $b \rightarrow \infty$, the second term becomes one. The first term represents the number of passengers at the bus stop $m$ with stopping, the second term represents the number of passengers taking the next bus $j$ -1 when bus $j$ passes over the bus stop $m$, and the third term represents the number of passengers taking the next bus $j$ when bus $j+1$ passes the bus stop $m$.

Furthermore, we take into account the speed up of a nonstopping bus. The speed up of the nonstopping bus is included in the operation velocity function as follows:

$$
\begin{aligned}
V\left(\Delta t_{j}(m)\right)= & v_{\min }+\frac{\left(v_{\max }-v_{\min }\right)}{4} \\
& \times\left[\tanh \left(\Delta t_{j}(m)-t_{c}\right)+\tanh \left(t_{c}\right)\right] \\
& \times\left\{1+\exp \left[-A\left(\Delta t_{j}(m), \Delta t_{j+1}(m)\right)\right]\right\} \\
& +c\left[1+\tanh \left\{b\left(\Delta t_{j}(m)-t_{s}\right)\right\}\right] .
\end{aligned}
$$

The third term of Eq. (8) represents the speed up when the delayed bus passes over the bus stop $m$ without stopping. If the time headway $\Delta t_{j}(m)$ of a delayed bus is larger than $t_{s}$, the bus passes over the bus stop $m$ without stopping and simultaneously increases speed by $c$. In the following, we consider the three versions of the time-headway model: (A) the basic model described by Eqs. (3), (4), and (5), (B) the 


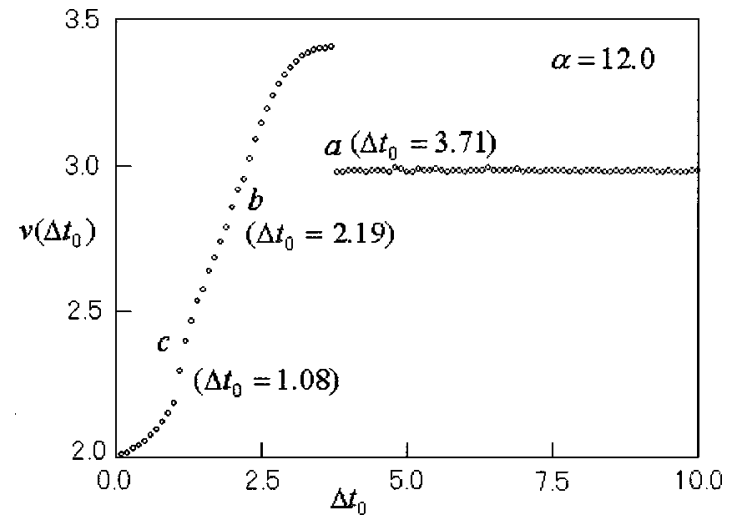

FIG. 3. Plot of the average velocity $v\left(\Delta t_{0}\right)$ as a function of the initial time headway $\Delta t_{0}$ for the basic version A of the timeheadway model where $\alpha=12.0, t_{c}=2.0, \lambda=0.2, v_{\min }=2.0$, and $v_{\max }=4.0$. The labels $a, b$, and $c$ indicate the transition points.

nonstopping model described by Eqs. (3), (4), (6), and (7), and $(\mathrm{C})$ the nonstopping and speed up model described by Eqs. (3), (4), (7), and (8).

\section{SIMULATION AND RESULT}

We carry out a computer simulation for the three versions of the bus route model. We solve Eqs. (3) and (4) by iteration under the periodic boundary condition. We assume that the distance between bus stops is the same for all bus stops: $L_{m}=\alpha$. The simulation is carried out for the following initial condition:

$$
\Delta t_{j}(0)=\Delta t_{0}+0.01 \times\{R(1)-0.5\},
$$

where $R(1)$ is the random number ranging from 0 to 1 and $\Delta t_{0}$ is the initial value of the time headway which is the same for all buses. The second term represents a small fluctuation of time headway at an initial stage. We study the dependence of bus velocity on the initial time headway. We plot in Fig. 3 the average velocity $v\left(\Delta t_{0}\right)$ at a steady state as a function of the initial time headway $\Delta t_{0}$ where $\alpha=12.0$, $t_{c}=2.0, \lambda=0.2, v_{\min }=2.0, v_{\max }=4.0$, and $N=100(N$ is the total number of buses). The average velocity at bus stop $m$ is defined as $v\left(\Delta t_{0}\right)=(1 / N) \sum_{j=1}^{N} V\left(\Delta t_{j}(m)\right)$ where $\Delta t_{0}$ $=(1 / N) \sum_{j=1}^{N} \Delta t_{j}(m)$. We find the three discontinuous points labeled by $a, b$, and $c$ in Fig. 3. The average velocity at a steady state increases with the time headway to $\Delta t_{0}=1.08$. At point $c$, the average velocity increases discontinuously by a small quantity. There is a small gap at point $c$ in the velocity curve. Then, the average velocity increases to point $b\left(\Delta t_{0}=2.19\right)$. At point $b$, there is a small gap in the velocity curve. Furthermore, the average velocity increases with time headway to point $a$. At point $a$ of $\Delta t_{0}=3.71$, the velocity decreases abruptly to $v=2.98$. In time headway larger than $\Delta t_{0}=3.71$, the steady-state velocity remains nearly constant $(v=2.98)$. We find that there are four distinct states for the four regions: (a) the bunching phase for $\Delta t_{0}>\Delta t_{a}$ (=3.71), (b) the homogeneous free phase for $\Delta t_{a} \geqslant \Delta t_{0}$ $>\Delta t_{b}(=2.19)$, (c) the kink jam phase for $\Delta t_{b} \geqslant \Delta t_{0}>\Delta t_{c}$ $(=1.08)$, and (d) the homogeneous congested phase for
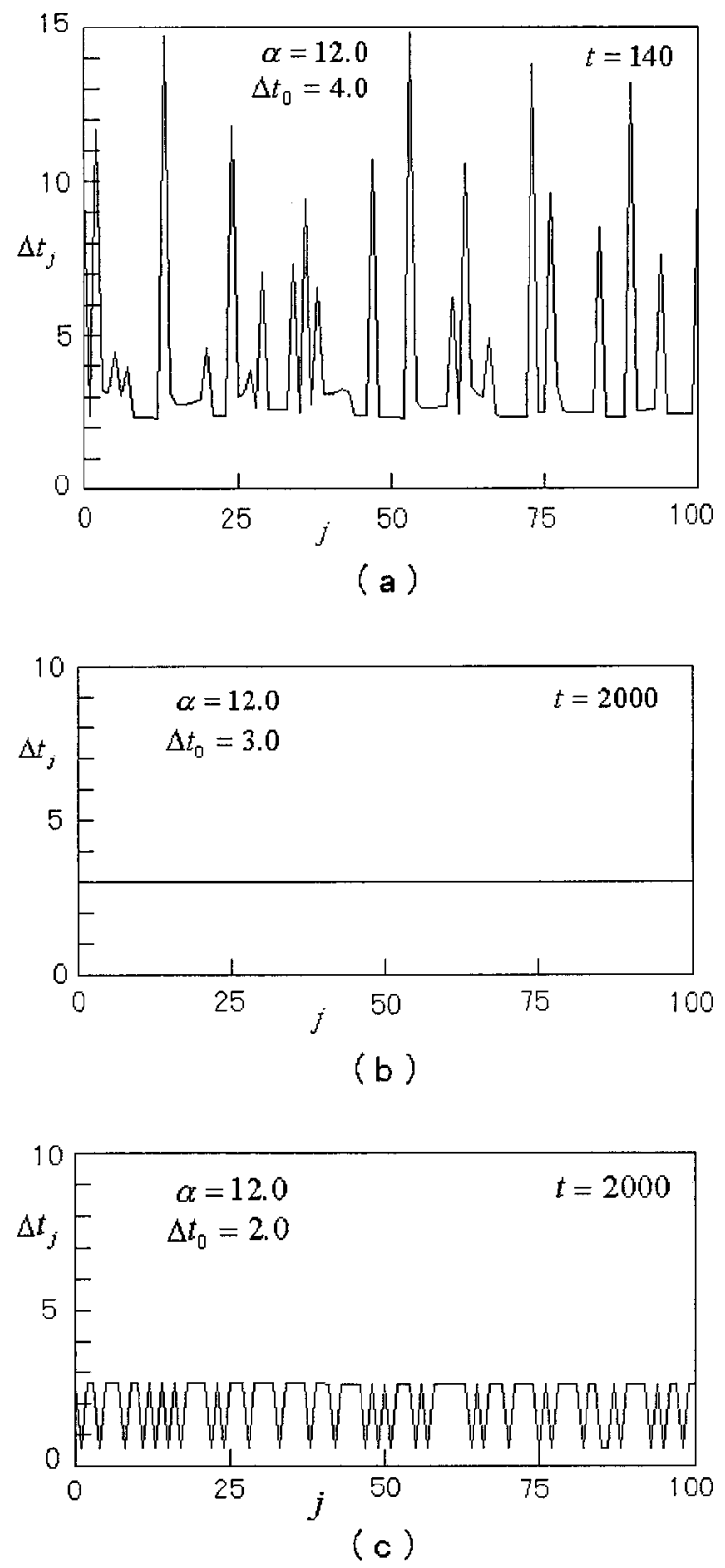

FIG. 4. The time-headway profiles $\Delta t_{j}$ plotted against bus number $j$ for the three distinct phases in version A where $\alpha=12.0, t_{c}$ $=2.0, \lambda=0.2, v_{\min }=2.0, v_{\max }=4.0$, and $N=100$. (a) The typical profile of an inhomogeneous bunching phase for $\Delta t_{0}=4.0$ and $t$ $=140$. (b) The typical profile of a homogeneous free phase for $\Delta t_{0}=3.0$ and $t=2000$. (c) The typical profile of a coexisting phase for $\Delta t_{0}=2.0$ and $t=2000$.

$\Delta t_{c} \geqslant \Delta t_{0}$. In Fig. 4 we show the time-headway profiles $\Delta t_{j}$ plotted against bus number $j$ for various values of initial time headway $\Delta t_{0}$ for $\alpha=12.0$ where the total number of buses is $N=100$ and $t$ is defined as $t=m+$ integer $\times M$. The profile (a) is obtained for $\Delta t_{0}=4.0$ and $t=140$. The profile (a) represents the typical pattern of the bunching phase. The time headways of some buses increases accordingly as the buses proceed. The buses behind a bus with large time headway have a small time headway. The profile (b) is obtained for $\Delta t_{0}=3.0$ and $t=2000$. The profile (b) represents the typical pattern of the homogeneous free phase. All the buses have 


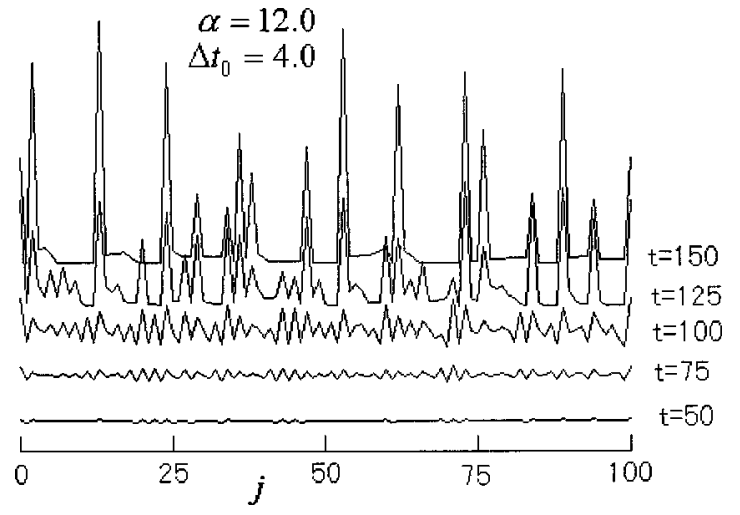

FIG. 5. Time evolution of the time-headway profile for the inhomogeneous bunching phase with the same values of parameters as in Fig. 4(a).

the same time headway as the initial value. The profile (c) is obtained for $\Delta t_{0}=2.0$ and $t=2000$. The profile (c) represents the typical pattern of the kink jam phase. The time headway oscillates from bus to bus. The profile is similar to the coexisting phase in the traffic flow. Figure 5 shows the time evolution of time headway for the bunching phase with the same values of parameters as Fig. 4(a). The fluctuation at an initial stage grows accordingly as buses proceed. The delayed buses catch up with the bus behind. In time, the time headways of some buses increases with time.

We study version B of the time-headway model. We use the operation velocity function (6) with (7). The buses pass over bus stops when the time headway becomes larger than $t_{s}=10.0$. In Fig. 6 we show the space-time plot of time headway from $t=100$ to $t=300$ for $\alpha=12.0, \Delta t_{0}=4.0$, and $t_{s}=10.0$ where $N=100$. Figure 7 shows the time-headway profile plotted against bus $j$ obtained at $t=300$ for the same values of parameters as in Fig. 6. The time headway of most delayed buses becomes less than the critical value $t_{s}=10.0$ but the time headway of some buses becomes larger than the critical value $t_{s}=10.0$. In the delayed bus with less time headway than $t_{s}=10.0$, the time headway decreases accordingly as the bus proceeds. However, in the delayed bus with larger time headway than $t_{s}=10.0$, the time headway in-

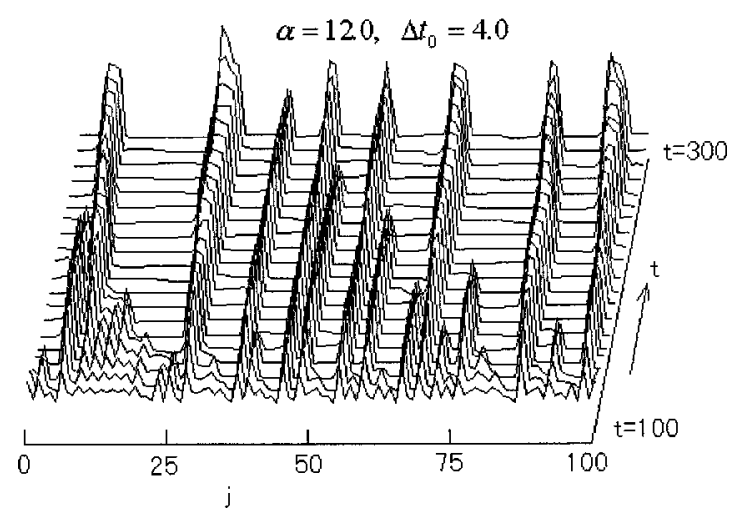

FIG. 6. Space-time plot of time headway from $t=100$ to $t$ $=300$ for version B where $\alpha=12.0, \Delta t_{0}=4.0$, and $t_{s}=10.0$, and $N=100$

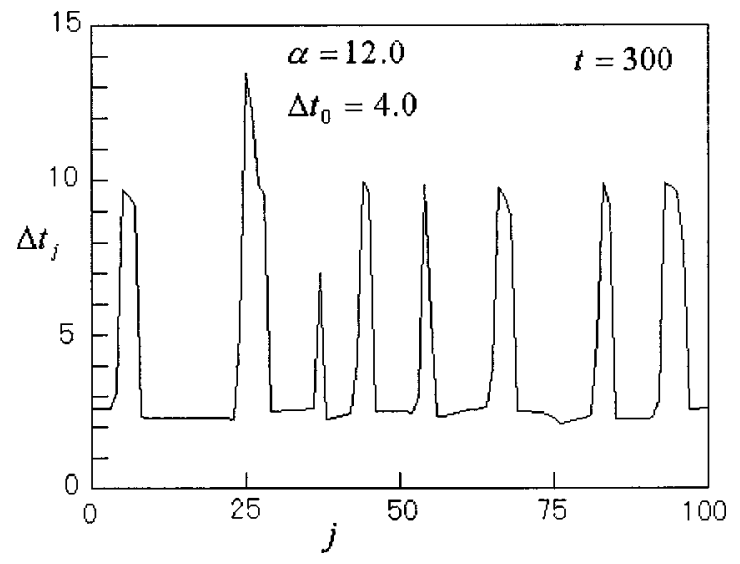

FIG. 7. Time-headway profile plotted against bus $j$ obtained at $t=300$ for the same values of parameters as in Fig. 6 .

creases accordingly as the bus proceeds. We compare Fig. 7 with Fig. 4(a). The nonstopping improves the delay of most delayed buses. However, some delayed buses become still more and more delayed. The bus driver should speed up in addition to the nonstopping to further reduce the delay.

In Fig. 8 we plot the average velocity $v\left(\Delta t_{0}\right)$ against the initial headway $\Delta t_{0}$ where $\alpha=12.0, t_{c}=2.0, t_{s}=10.0$, and $N=100$. We compare Fig. 8 with Fig. 3 . The bunching transitions occur at the same values as in Fig. 3. In the bunching phase, the bus velocity is larger than that in Fig. 3. The increase of bus velocity is due to the nonstopping effect. The transition points do not change by introducing the nonstopping effect. In the homogeneous free phase, the coexisting phase, and the homogeneous congested phase, the timeheadway profiles do not change and are the same as those of version A.

We study version $\mathrm{C}$ of the time-headway model. We use the operation velocity function (8) with (7). The buses pass over bus stops when the time headway becomes larger than $t_{s}=10.0$ and simultaneously speeds up. In Fig. 9, we show the space-time plot of time headway from $t=50$ to $t=225$ for $\alpha=12.0, \Delta t_{0}=4.0$, and $t_{s}=10.0$ where $c=1.0$ and $N$ $=100$. The values of the parameters are the same as those of version B in Fig. 6. Figure 10 shows the time-headway pro-

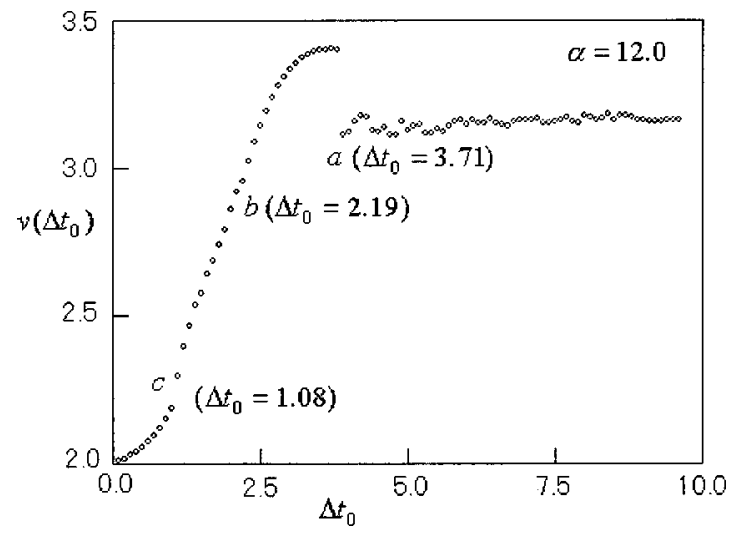

FIG. 8. Plot of average velocity $v\left(\Delta t_{0}\right)$ against the initial headway $\Delta t_{0}$ for version B where $\alpha=12.0, t_{c}=2.0, t_{s}=10.0$, and $N$ $=100$. 


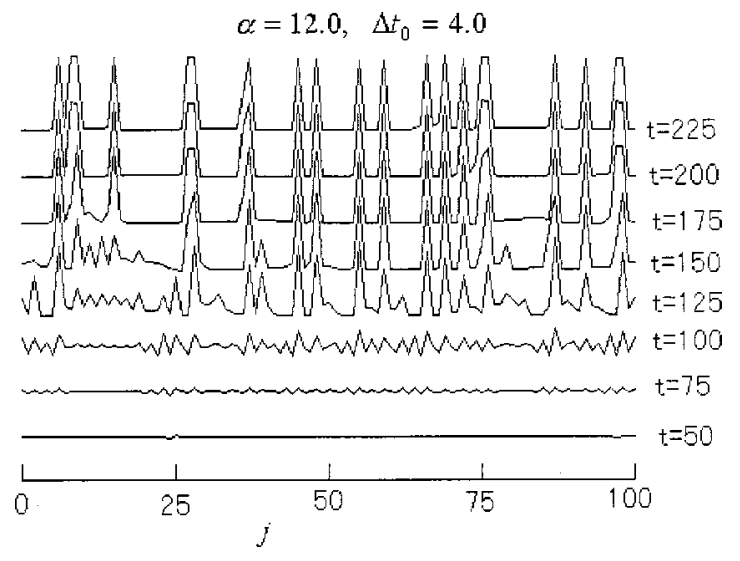

FIG. 9. Space-time plot of time headway from $t=50$ to $t$ $=225$ for version $\mathrm{C}$ where $\alpha=12.0, \Delta t_{0}=4.0, t_{s}=10.0, c=1.0$, and $N=100$.

file plotted against bus $j$ obtained at $t=2500$ for the same values of parameters as in Fig. 9. After $t=200$, the timeheadway profile does not change and the flow of buses is in a steady state. The delayed buses move with a constant time headway, $\Delta t=9.2$ and its time headway is a little less than the critical value $t_{s}=10.0$. The normal buses behind the delayed buses move with a constant time headway, $\Delta t=2.6$. The time headway of all the buses is less than $t_{s}=10.0$. We compare Fig. 10 with Fig. 4(a). The nonstopping and speed up improves the delay of all delayed buses.

In Fig. 11 we plot the average velocity $v\left(\Delta t_{0}\right)$ against the initial headway $\Delta t_{0}$ where $\alpha=12.0, t_{c}=2.0, t_{s}=10.0, c$ $=1.0$, and $N=100$. We compare Fig. 11 with Figs. 3 and 8 . The bunching transitions occur at the same values as in Figs. 3 and 8 . In the bunching phase, the bus velocity is larger than those in Figs. 3 and 8. The increase of the bus velocity is due to both nonstopping and speed-up effects. The transition points do not change by introducing both nonstopping and speed-up effects. In the homogeneous free phase, the coexisting phase, and the homogeneous congested phase, the time-headway profiles do not change and are the same as those of versions A and B. Thus we find that the buses are operated correctly by both nonstopping and speed up of the delayed buses. There are no delayed buses with larger time headway than $t_{s}=10.0$.

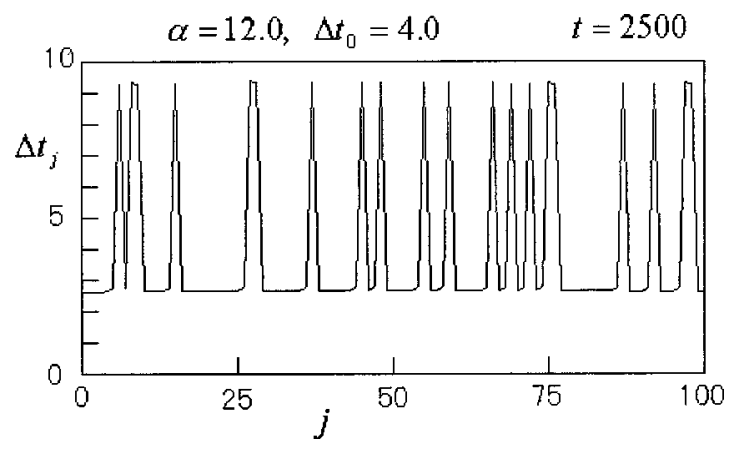

FIG. 10. Time-headway profile of version $\mathrm{C}$ plotted against bus $j$ obtained at $t=2500$ for the same values of parameters as in Fig. 9.

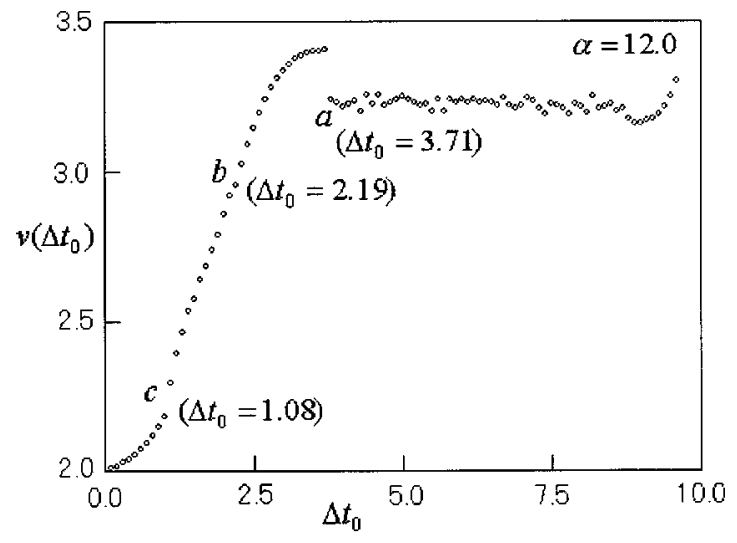

FIG. 11. Plot of the average velocity $v\left(\Delta t_{0}\right)$ against the initial headway $\Delta t_{0}$ for version $\mathrm{C}$ where $\alpha=12.0, t_{c}=2.0, t_{s}=10.0, c$ $=1.0$, and $N=100$.

\section{LINEAR STABILITY ANALYSIS}

We consider the stability of a homogeneous flow of buses. The homogeneous flow is defined by such a state that all buses move with the same time headway $\Delta t_{0}$ and the same operation velocity $V\left(\Delta t_{0}\right)$. We apply the linear stability analysis to our model (3) with the operation velocity (4). Let $y_{j}(m)$ be small deviations from the homogeneous flow: $\Delta t_{j}(m)=\Delta t_{0}+y_{j}(m)$. Then, the linear equation is obtained from Eqs. (3) and (4)

$$
y_{j}(m)=y_{j}(m-1)+\frac{\alpha V^{\prime}\left(\Delta t_{0}\right)}{V\left(\Delta t_{0}\right)^{2}}\left\{y_{j+1}(m-1)-y_{j}(m-1)\right\},
$$

where $\alpha=L_{m}$ and $V^{\prime}\left(\Delta t_{0}\right)$ is the derivative of operation velocity $V(x)$ at $x=\Delta t_{0}$ :

$$
V^{\prime}\left(\Delta t_{0}\right)=\left.\frac{d V(x)}{d x}\right|_{x=\Delta t_{0}} .
$$

By expanding $y_{j}(m)=Y \exp (i k j+z m)$, one obtains

$$
e^{z}=1+\frac{\alpha V^{\prime}\left(\Delta t_{0}\right)}{V\left(\Delta t_{0}\right)^{2}}\left(e^{i k}-1\right) .
$$

By solving Eq. (11) with $z$, one finds that the leading term of $z$ is an order of $i k$. When $i k \rightarrow 0, z \rightarrow 0$. Let us derive the long wave expansion of $z$, which is determined order by order around $i k \approx 0$. By expanding $z=z_{1} i k+z_{2}(i k)^{2}+\cdots$, the first-order and second-order terms of $i k$ are obtained,

$$
\begin{gathered}
z_{1}=\frac{\alpha V^{\prime}\left(\Delta t_{0}\right)}{V\left(\Delta t_{0}\right)^{2}}, \\
z_{2}=\frac{\alpha V^{\prime}\left(\Delta t_{0}\right)}{2 V\left(\Delta t_{0}\right)^{2}}\left\{1-\frac{\alpha V^{\prime}\left(\Delta t_{0}\right)}{V\left(\Delta t_{0}\right)^{2}}\right\} .
\end{gathered}
$$

If $z_{2}$ is a negative value, the homogeneous flow becomes unstable for long wavelength modes. When $z_{2}$ is a positive 


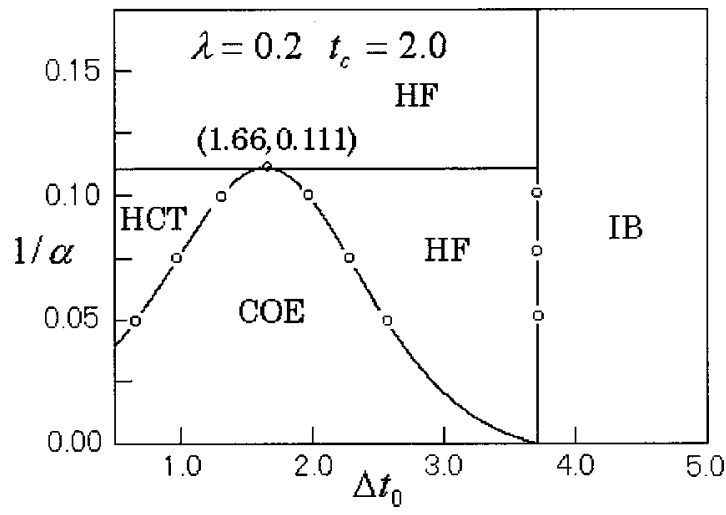

FIG. 12. Phase diagram in the space $\left(\Delta t_{0}, 1 / \alpha\right)$ for $\lambda=0.2$, $v_{\min }=2.0, v_{\max }=4.0$, and $t_{c}=2.0$. The solid lines divide the space into four distinct phases: (1) the inhomogeneous bunching phase (IB), (2) the homogeneous free phase (HF), (3) the coexisting phase (COE), and (4) the homogeneous congested phase (HCT). The open circles indicate the transition points obtained by the simulation.

value, the homogeneous flow is stable. One finds the unstability condition for small disturbances of long wavelengths:

$$
V^{\prime}\left(\Delta t_{0}\right)<0 \text {, }
$$

or

$$
\alpha>\frac{V\left(\Delta t_{0}\right)^{2}}{V^{\prime}\left(\Delta t_{0}\right)} \text { for } V\left(\Delta t_{0}\right)>0 .
$$

The unstability condition (15) is similar to that of the conventional car-following model for traffic [27]. The neutral stability line $\alpha=V\left(\Delta t_{0}\right)^{2} / V^{\prime}\left(\Delta t_{0}\right)$ for $V\left(\Delta t_{0}\right)>0$ presents the jamming transition curve among the homogeneous free phase (HF), the coexisting phase (COE), and the homogeneous congested phase (HCT). The unstability condition (14) is a new one and occurs due to the delay effect of a bus for a large gap. The neutral stability condition $V^{\prime}\left(\Delta t_{0}\right)=0$ presents the transition line between the inhomogeneous bunching phase (IB) and the HF. Figure 12 shows the phase diagram in the space $\left(\Delta t_{0}, 1 / \alpha\right)$ for $\lambda=0.2, v_{\min }=2.0, v_{\max }$ $=4.0$, and $t_{c}=2.0$. The solid curve is given by $\alpha$ $=V\left(\Delta t_{0}\right)^{2} / V^{\prime}\left(\Delta t_{0}\right)$ for $V\left(\Delta t_{0}\right)>0$. The vertical line is

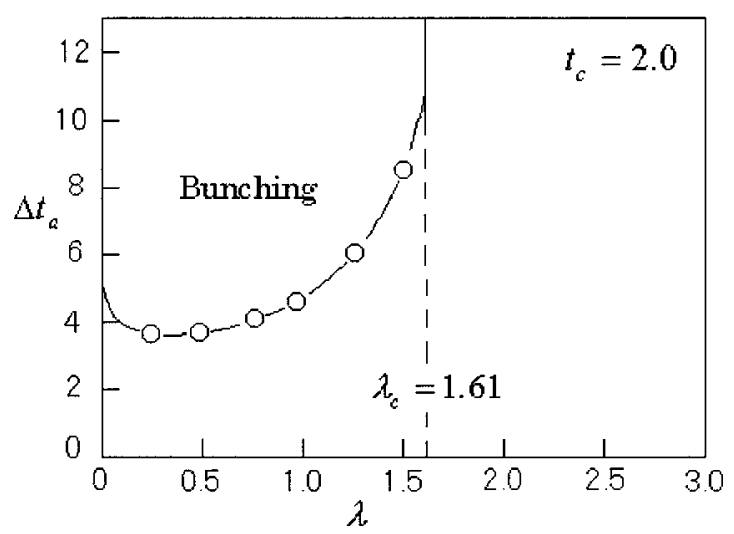

FIG. 13. Plot of the bunching transition line $V^{\prime}\left(\Delta t_{0}\right)=0$ against $\lambda$ for $t_{c}=2.0$. The inhomogeneous bunching phase appears above the solid line. The open circles indicate the simulation result.

given by $V^{\prime}\left(\Delta t_{0}\right)=0$. The simulation result is indicated by open circles. The simulation result agrees with the neutral stability lines obtained from the linear stability analysis. The operation velocity has the maximal point for $\lambda<1.61$. The bunching transition does not occur for $\lambda>1.61$ since the operation velocity function increases monotonically for $\lambda$ $>1.61$. In Fig. 13, we show the plot of the bunching transition line $V^{\prime}\left(\Delta t_{0}\right)=0$ against $\lambda$ for $t_{c}=2.0$. The inhomogeneous bunching phase appears above the solid line. The open circles indicate the simulation result. The theoretical curve obtained by the linear stability analysis is consistent with the simulation result.

\section{SUMMARY}

We have presented the time-headway model for a bus route. We have investigated bus behavior and the bunching transition. We have shown that the dynamical phase transitions occurs with varying the initial time headway. We have also applied the linear stability analysis to our model. We have derived the neutral stability curves which give the dynamical phase transition lines. We have found that the neutral stability curves agree with the simulation result.

It will be useful to investigate bus behavior in the route with the use of the time headway model proposed here.
[1] D. Chowdhury, L. Santen, and A. Schadschneider, Phys. Rep. 329, 199 (2000).

[2] Traffic and Granular Flow, edited by D. E. Wolf, M. Schreckenberg, and A. Bachem (World Scientific, Singapore, 1996).

[3] D. Helbing, Verkehrsdynamik (Springer, Berlin, 1997).

[4] Traffic and Granular Flow 97, edited by M. Schreckenberg and D. E. Wolf (Springer, Singapore, 1998).

[5] G. F. Newell, Oper. Res. 9, 209 (1961).

[6] G. B. Whitham, Proc. R. Soc. London, Ser. A 428, 49 (1990).

[7] K. Nagel and M. Schreckenberg, J. Phys. I 2, 2221 (1992).

[8] M. Bando, K. Hasebe, A. Nakayama, A. Shibata, and Y. Sugiyama, Phys. Rev. E 51, 1035 (1995).

[9] B. S. Kerner, P. Konhauser, and M. Schilke, Phys. Rev. E 51, 6243 (1995).
[10] B. S. Kerner and H. Rehborn, Phys. Rev. E 53, R1297 (1996).

[11] O. Biham, A. A. Middleton, and D. A. Levine, Phys. Rev. A 46, R6124 (1992).

[12] T. Nagatani, Phys. Rev. E 48, 3290 (1993).

[13] J. A. Cuesta, F. C. Matinez, J. M. Nolera, and A. Sanchez, Phys. Rev. E 48, 4175 (1993).

[14] K. Nagel, D. E. Wolf, P. Wagner, and P. Simon, Phys. Rev. E 58, 1425 (1998).

[15] D. Helbing and M. Schreckenberg, Phys. Rev. E 59, R2505 (1999).

[16] D. Helbing, Phys. Rev. E 53, 2366 (1996).

[17] M. Treiber, A. Hennecke, and D. Helbing, Phys. Rev. E 59, 239 (1999). 
[18] K. Nagel, Phys. Rev. E 53, 4655 (1996).

[19] S. Krauss, P. Wagner, and C. Gawron, Phys. Rev. E 55, 5597 (1997).

[20] T. Komatsu and S. Sasa, Phys. Rev. E 52, 5574 (1995).

[21] T. Nagatani and K. Nakanishi, Phys. Rev. E 57, 6415 (1998).

[22] T. Nagatani, K. Nakanishi, and H. Emmerich, J. Phys. A 31, 5431 (1998).

[23] D. Helbing, A. Hennecke, and M. Treiber, Phys. Rev. Lett. 82, 4360 (1999).
[24] H. Y. Lee, H. W. Lee, and D. Kim, Phys. Rev. E 59, 5101 (1999).

[25] B. S. Kerner, P. Konhauser, and M. Schilke, Phys. Lett. A 215 , 45 (1996).

[26] D. Helbing and M. Treiber, Phys. Rev. Lett. 81, 3042 (1998).

[27] T. Nagatani, Phys. Rev. E 58, 4271 (1998).

[28] T. Nagatani, Phys. Rev. E 59, 4857 (1999).

[29] O. J. O'loan, M. R. Evans, and M. E. Cates, Phys. Rev. E 58, 1404 (1998). 